\title{
CRITICAL GROUPS OF CRITICAL POINTS PRODUCED BY LOCAL LINKING WITH APPLICATIONS
}

\author{
KANISHKA PERERA
}

ABSTRACT. We prove the existence of nontrivial critical points with nontrivial critical groups for functionals with a local linking at 0 . Applications to elliptic boundary value problems are given.

\section{INTRODUCTION}

Let $F$ be a real $C^{1}$ function defined on a Banach space $X$. We say that $F$ has a local linking near the origin if $X$ has a direct sum decomposition $X=X_{1} \oplus X_{2}$ with $\operatorname{dim} X_{1}<\infty, F(0)=0$, and, for some $r>0$,

$$
\left\{\begin{array}{l}
F(u) \leq 0 \text { for } u \in X_{1},\|u\| \leq r, \\
F(u)>0 \text { for } u \in X_{2}, 0<\|u\| \leq r .
\end{array}\right.
$$

Then it is clear that 0 is a critical point of $F$.

The notion of local linking was introduced by Li and Liu [7], [8], who proved the existence of nontrivial critical points under various assumptions on the behavior of $F$ at infinity. These results were recently generalized by Brézis and Nirenberg [3], Li and Willem [9], and several other authors.

In infinite dimensional Morse theory (see Chang [5] or Mawhin and Willem [11]), the local behavior of $F$ near an isolated critical point $u_{0}$, $F\left(u_{0}\right)=c$, is described by the sequence of critical groups

$$
C_{q}\left(F, u_{0}\right)=H_{q}\left(F_{c} \cap U,\left(F_{c} \cap U\right) \backslash\left\{u_{0}\right\}\right) \quad q \in \mathbb{Z}
$$

where $F_{c}$ is the sublevel set $\{u \in X: F(u) \leq c\}, U$ is a neighborhood of $u_{0}$ such that $u_{0}$ is the only critical point of $F$ in $F_{c} \cap U$, and $H_{*}(\cdot, \cdot)$ denote the singular relative homology groups.

1991 Mathematics Subject Classification. Primary 58E05.

Key words and phrases. Morse theory, critical groups, local linking.

Received: March 10, 1998. 
It was proved in Liu [10] that if $F$ has a local linking near the origin, $\operatorname{dim} X_{1}=j$, and 0 is an isolated critical point of $F$, then $C_{j}(F, 0) \neq 0$. In the present paper we use this fact to obtain a nontrivial critical point $u$ with either $C_{j+1}(F, u) \neq 0$ or $C_{j-1}(F, u) \neq 0$. When $X$ is a Hilbert space and $F$ is $C^{2}$, this yields Morse index estimates for $u$ via the Shifting theorem.

When $X$ is a Hilbert space and $d F$ is Lipschitz in a neighborhood of the origin, we extend the result of Liu $[10]$ to the case where $F$ satisfies the "relaxed" local linking condition

$$
\left\{\begin{array}{l}
F(u) \leq 0 \text { for } u \in X_{1},\|u\| \leq r, \\
F(u) \geq 0 \text { for } u \in X_{2},\|u\| \leq r
\end{array}\right.
$$

(see Brézis and Nirenberg [3] and Li and Willem [9]), and thus obtain a nontrivial critical point with a nontrivial critical group in this case also.

We apply our abstract result to elliptic boundary value problems, including an equation asymptotically linear at $-\infty$ and superlinear at $+\infty$, and prove new multiplicity results.

\section{Abstract Result}

Throughout this section we assume that $F$ satisfies the Palais-Smale compactness condition (PS) and has only isolated critical values, with each critical value corresponding to a finite number of critical points.

Theorem 2.1. Suppose that there is a critical point $u_{0}$ of $F, F\left(u_{0}\right)=c$, with $C_{j}\left(F, u_{0}\right) \neq 0$ for some $j \geq 0$ and regular values $a, b$ of $F, a<c<b$, such that $H_{j}\left(F_{b}, F_{a}\right)=0$. Then $F$ has a critical point $u$ with either

$$
\begin{gathered}
c<F(u)<b \text { and } C_{j+1}(F, u) \neq 0, \text { or } \\
a<F(u)<c \text { and } C_{j-1}(F, u) \neq 0 .
\end{gathered}
$$

Proof of Theorem 2.1 makes use of the following topological lemma:

Lemma 2.2. If $B^{\prime} \subset B \subset A \subset A^{\prime}$ are topological spaces such that $H_{j}(A, B) \neq 0$ and $H_{j}\left(A^{\prime}, B^{\prime}\right)=0$, then either

$$
H_{j+1}\left(A^{\prime}, A\right) \neq 0 \text { or } H_{j-1}\left(B, B^{\prime}\right) \neq 0 .
$$

Proof. Suppose that $H_{j+1}\left(A^{\prime}, A\right)=0$. Since $H_{j}\left(A^{\prime}, B^{\prime}\right)$ is also trivial, it follows from the following portion of the exact sequence of the triple $\left(A^{\prime}, A, B^{\prime}\right)$ that $H_{j}\left(A, B^{\prime}\right)=0$ :

$$
H_{j+1}\left(A^{\prime}, A\right) \stackrel{\partial_{*}}{\longrightarrow} H_{j}\left(A, B^{\prime}\right) \stackrel{i_{*}}{\longrightarrow} H_{j}\left(A^{\prime}, B^{\prime}\right)
$$

Since $H_{j}(A, B) \neq 0$, now it follows from the following portion of the exact sequence of the triple $\left(A, B, B^{\prime}\right)$ that $H_{j-1}\left(B, B^{\prime}\right) \neq 0$ :

$$
H_{j}\left(A, B^{\prime}\right) \stackrel{j_{*}}{\longrightarrow} H_{j}(A, B) \stackrel{\partial_{*}}{\longrightarrow} H_{j-1}\left(B, B^{\prime}\right) \square
$$


Proof of Theorem 2.1. Take $\epsilon, 0<\epsilon<\min \{c-a, b-c\}$ such that $c$ is the only critical value of $F$ in $[c-\epsilon, c+\epsilon]$. Then, since $C_{j}\left(F, u_{0}\right) \neq 0$, it follows from Chapter I, Theorem 4.2 of Chang [5] that $H_{j}\left(F_{c+\epsilon}, F_{c-\epsilon}\right) \neq$ 0 . Since $H_{j}\left(F_{b}, F_{a}\right)=0$, by Lemma 2.2 , either $H_{j+1}\left(F_{b}, F_{c+\epsilon}\right) \neq 0$ or $H_{j-1}\left(F_{c-\epsilon}, F_{a}\right) \neq 0$, and the conclusion follows from Chapter I, Theorem 4.3 and Corollary 4.1 of Chang [5].

As mentioned before, if $F$ has a local linking near the origin, $\operatorname{dim} X_{1}=$ $j$, then $C_{j}(F, 0) \neq 0$ (see Liu [10]), and hence the following corollary is immediate from Theorem 2.1:

Corollary 2.3. Suppose $F$ has a local linking near the origin, $\operatorname{dim} X_{1}=j$. Assume also that there are regular values $a, b$ of $F, a<0<b$, such that $H_{j}\left(F_{b}, F_{a}\right)=0$. Then $F$ has a critical point $u$ with either

$$
\begin{gathered}
0<F(u)<b \text { and } C_{j+1}(F, u) \neq 0, \text { or } \\
a<F(u)<0 \text { and } C_{j-1}(F, u) \neq 0 .
\end{gathered}
$$

If $X$ is a Hilbert space, $F$ is $C^{2}$, and $u$ is a critical point of $F$, we denote by $m(u)$ the Morse index of $u$ and by $m^{*}(u)=m(u)+\operatorname{dim} \operatorname{ker} d^{2} F(u)$ the large Morse index of $u$. We recall that if $u$ is nondegenerate and $C_{q}(F, u) \neq 0$, then $m(u)=q$ (see Chapter I, Theorem 4.1 of Chang [5]). Let us also recall that it follows from the Shifting theorem (Chapter I, Theorem 5.4 of Chang [5]) that if $u$ is degenerate, 0 is an isolated point of the spectrum of $d^{2} F(u)$, and $C_{q}(F, u) \neq 0$, then $m(u) \leq q \leq m^{*}(u)$. Hence we have the following corollary:

Corollary 2.4. Let $X$ be a Hilbert space and $F$ be $C^{2}$ in Theorem 2.1. Assume that for every degenerate critical point $u$ of $F, 0$ is an isolated point of the spectrum of $d^{2} F(u)$. Then $F$ has a critical point $u$ with either

$$
\begin{gathered}
c<F(u)<b \text { and } m(u) \leq j+1 \leq m^{*}(u), \text { or } \\
a<F(u)<c \text { and } m(u) \leq j-1 \leq m^{*}(u) .
\end{gathered}
$$

Remark 2.5. In particular, Corollary 2.4 yields a critical point $u \neq u_{0}$ with $m(u) \leq j+1$ and $j-1 \leq m^{*}(u)$. Benci and Fortunato [2] have proved this fact for the special case where $u_{0}$ is a nondegenerate critical point with Morse index $j$, but without assuming that the critical points of $F$ are isolated. Their proof is based on a generalized Morse theory due to Benci and Giannoni [1]. However, Corollary 2.4 says, in addition, that $u$ is at a level different from $F\left(u_{0}\right)$.

If $X$ is a Hilbert space and $d F$ is Lipschitz in a neighborhood of the origin, we can relax the local linking condition as in (2). This follows from the following extension of the result of Liu [10] (see also Theorem 5.6 of Kryszewski and Szulkin [6]):

Theorem 2.6. Let $X$ be a Hilbert space and $d F$ be Lipschitz in a neighborhood of the origin. Suppose that $F$ satisfies the local linking condition (2), $\operatorname{dim} X_{1}=j$. Then $C_{j}(F, 0) \neq 0$. 
Our proof of Theorem 2.6 uses the following "deformation" lemma:

Lemma 2.7. Under the assumptions of Theorem 2.6 there exist a closed ball $B$ centered at the origin and a homeomorphism $h$ of $X$ onto $X$ such that

1. 0 is the only critical point of $F$ in $h(B)$,

2. $\left.h\right|_{B \cap X_{1}}=i d_{B \cap X_{1}}$,

3. $F(u)>0$ for $u \in h\left(B \cap X_{2} \backslash\{0\}\right)$.

Proof. Take open balls $B^{\prime}, B^{\prime \prime}$ centered at the origin, with $\overline{B^{\prime}} \subset B^{\prime \prime}$, such that 0 is the only critical point of $F$ in $B^{\prime}$ and $d F$ is Lipschitz in $B^{\prime \prime}$, and let $B \subset B^{\prime}$ be a closed ball centered at the origin with radius $\leq r$ (in (2)). Since $B$ and $\left(B^{\prime}\right)^{c}$ are disjoint closed sets there is a locally Lipschitz nonnegative function $g \leq 1$ satisfying

$$
g= \begin{cases}1 & \text { on } B \\ 0 & \text { outside } B^{\prime}\end{cases}
$$

Consider the vector field

$$
V(u)=g(u)\|P u\| d F(u)
$$

where $P$ is the orthogonal projection onto $X_{2}$. Clearly $V$ is locally Lipshitz and bounded on $X$. Consider the flow $\eta(t)=\eta(t, u)$ defined by

$$
\frac{d \eta}{d t}=V(\eta),\left.\eta\right|_{t=0}=u \text {. }
$$

Clearly, $\eta$ is defined for $t \in[0,1]$. Let $h=\eta(1, \cdot)$. Since $\left.h\right|_{\left(B^{\prime}\right)^{c}}=\operatorname{id}_{\left(B^{\prime}\right)^{c}}$ and $h$ is one-to-one, $h(B) \subset B^{\prime}$ and 1 follows. For $u \in B \cap X_{2} \backslash\{0\}$,

$$
F(h(u))=F(u)+\int_{0}^{1} g(\eta(t))\|P \eta(t)\|\|d F(\eta(t))\|^{2} d t>0
$$

since $F(u) \geq 0$ and $g(u)\|P u\|\|d F(u)\|^{2}>0$.

Proof of Theorem 2.6. By 1 of Lemma 2.7, $C_{j}(F, 0)=H_{j}\left(F_{0} \cap h(B), F_{0} \cap\right.$ $h(B) \backslash\{0\})$.

By the local linking condition (2) and 2 and 3 of Lemma 2.7, $\partial B \cap X_{1} \subset$ $F_{0} \cap h(B) \backslash\{0\} \subset h\left(B \backslash X_{2}\right)$ and $B \cap X_{1} \subset F_{0} \cap h(B)$. Since $\left.h\right|_{\partial B \cap X_{1}}=\operatorname{id} d_{\partial B \cap X_{1}}$, the inclusion $\partial B \cap X_{1} \hookrightarrow h\left(B \backslash X_{2}\right)$ can also be written as the composition of the inclusion $\partial B \cap X_{1} \stackrel{i^{\prime}}{\hookrightarrow} B \backslash X_{2}$ and the restriction of $h$ to $B \backslash X_{2}$. Hence we have the following commutative diagram induced by inclusions and $h$ :

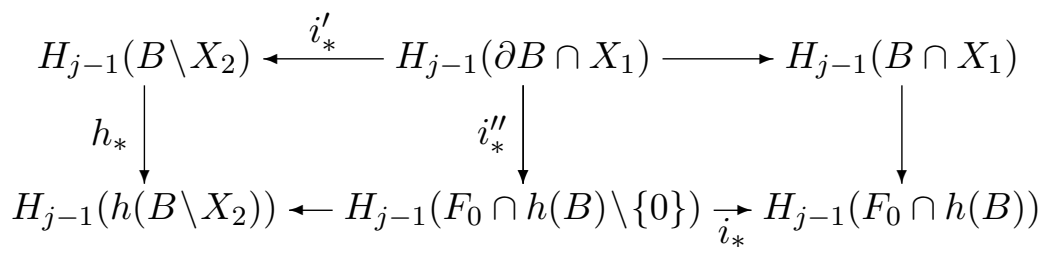

Since $\partial B \cap X_{1}$ is a strong deformation retract of $B \backslash X_{2}$ and $h$ is a homeomorphism, $i_{*}^{\prime}$ and $h_{*}$ are isomorphisms and hence $i_{*}^{\prime \prime}$ is a monomorphism. 
Since $\operatorname{rank} H_{j-1}\left(B \cap X_{1}\right)<\operatorname{rank} H_{j-1}\left(\partial B \cap X_{1}\right)$, then it follows that $i_{*}$ is not a monomorphism.

Now it follows from the following portion of the exact sequence of the pair $\left(F_{0} \cap h(B), F_{0} \cap h(B) \backslash\{0\}\right)$ that $C_{j}(F, 0)=H_{j}\left(F_{0} \cap h(B), F_{0} \cap h(B) \backslash\{0\}\right) \neq 0$ :

$$
C_{j}(F, 0) \stackrel{\partial_{*}}{\longrightarrow} H_{j-1}\left(F_{0} \cap h(B) \backslash\{0\}\right) \stackrel{i_{*}}{\rightarrow} H_{j-1}\left(F_{0} \cap h(B)\right)
$$

\section{Elliptic Boundary Value Problems}

Consider the problem

$$
\left\{\begin{aligned}
-\Delta u & =g(u) & & \text { in } \Omega, \\
u & =0 & & \text { on } \partial \Omega
\end{aligned}\right.
$$

where $\Omega$ is a bounded domain in $\mathbb{R}^{\ltimes}$ with smooth boundary $\partial \Omega$ and $g \in$ $C^{1}(\mathbb{R}, \mathbb{R})$ satisfies

$\left(g_{1}\right):|g(u)| \leq C\left(1+|u|^{p-1}\right)$ with $2<p<\frac{2 n}{n-2}$, for some $C>0$,

$\left(g_{2}\right): g(0)=0=g(a)$ for some $a>0$,

$\left(g_{3}\right)$ : there are constants $\mu>2$ and $A>0$ such that

$$
0<\mu G(u) \leq u g(u) \text { for }|u| \geq A,
$$

where $G(u):=\int_{0}^{u} g(t) d t$.

Let $\lambda=g^{\prime}(0)$ and let $0<\lambda_{1} \leq \lambda_{2} \leq \lambda_{3} \leq \cdots$ be the eigenvalues of $-\Delta$ with Dirichlet boundary condition.

Theorem 3.1. Assume that $g$ satisfies $\left(g_{1}\right)-\left(g_{3}\right)$ and one of the following conditions:

1. $\lambda_{j}<\lambda<\lambda_{j+1}$,

2. $\lambda_{j}=\lambda<\lambda_{j+1}$ and, for some $\delta>0$,

$$
G(u) \geq \frac{1}{2} \lambda u^{2} \text { for }|u| \leq \delta,
$$

3. $\lambda_{j}<\lambda=\lambda_{j+1}$ and, for some $\delta>0$,

$$
G(u) \leq \frac{1}{2} \lambda u^{2} \text { for }|u| \leq \delta .
$$

If $j \geq 3$, problem (3) has at least four nontrivial solutions.

Proof. Solutions of (3) are the critical points of the $C^{2}$ functional

$$
F(u)=\int_{\Omega} \frac{1}{2}|\nabla u|^{2}-G(u)
$$

defined on $X=H_{0}^{1}(\Omega)$. It is well known that $F$ satisfies (PS).

By a standard argument involving a cut-off technique and the strong maximum principle, $F$ has a local minimizer $u_{0}$ with $0<u_{0}<a$,

$$
\operatorname{rank} C_{q}\left(F, u_{0}\right)=\delta_{q 0} .
$$


Since $\lim _{t \rightarrow \infty} F\left( \pm t \phi_{1}\right)=-\infty$, where $\phi_{1}>0$ is the first Dirichlet eigenfunction of $-\Delta$, then $F$ also has two mountain pass points $u_{1}^{ \pm}$with $u_{1}^{-}<u_{0}<u_{1}^{+}$,

$$
\operatorname{rank} C_{q}\left(F, u_{1}^{ \pm}\right)=\delta_{q 1}
$$

(see the proof of Theorem B in Chang, Li, and Liu [4]).

Let $X_{1}$ be the $j$-dimensional space spanned by the eigenfunctions corresponding to $\lambda_{1}, \cdots, \lambda_{j}$ and let $X_{2}$ be its orthogonal complement in $X$. Then $F$ has a local linking near the origin with respect to the decomposition $X=X_{1} \oplus X_{2}$ (see the proof of Theorem 4 in Li and Willem [9]) and hence

$$
C_{j}(F, 0) \neq 0 \text {. }
$$

Also, for $\alpha<0$ and $|\alpha|$ sufficiently large,

$$
H_{q}\left(X, F_{\alpha}\right)=0 \quad \forall q \in \mathbb{Z}
$$

(see Lemma 3.2 of Wang [13]). Therefore, by Theorem 2.1, $F$ has a nontrivial critical point $u_{j}$ with either

$$
C_{j+1}\left(F, u_{j}\right) \neq 0 \text { or } C_{j-1}\left(F, u_{j}\right) \neq 0 .
$$

Since $j \geq 3$, a comparison of the critical groups shows that $u_{0}, u_{1}^{ \pm}, u_{j}$ are distinct nontrivial critical points of $F$.

Next we consider the following asymmetric problem of the AmbrosettiProdi type

$$
\left\{\begin{aligned}
-\Delta u+a(x) u & =g(x, u) & & \text { in } \Omega, \\
u & =0 & & \text { on } \partial \Omega
\end{aligned}\right.
$$

where $a \in L^{\infty}(\Omega)$ and $g \in C^{1}(\bar{\Omega} \times \mathbb{R}, \mathbb{R})$ satisfies

$\left(g_{1}\right):|g(x, u)| \leq C\left(1+|u|^{p-1}\right)$ with $2<p<\frac{2 n}{n-2}$, for some $C>0$,

$\left(g_{2}\right): g(x, 0)=g_{u}(x, 0)=0$,

$\left(g_{3}\right): \lim _{u \rightarrow-\infty} \frac{g(x, u)}{u}<\lambda_{1}$, uniformly in $\bar{\Omega}$,

$\left(g_{4}\right): \varlimsup_{u \rightarrow-\infty}\left(G(x, u)-\frac{1}{2} u g(x, u)\right)<+\infty$, uniformly in $\bar{\Omega}$,

$\left(g_{5}\right)$ : there are $\mu>2$ and $A>0$ such that

$$
0<\mu G(x, u) \leq u g(x, u) \text { for } u \geq A,
$$

where $G(x, u):=\int_{0}^{u} g(x, t) d t$.

Here $\lambda_{1}<\lambda_{2} \leq \lambda_{3} \leq \cdots$ denote the eigenvalues of $-\Delta+a$ with Dirichlet boundary condition.

Theorem 3.2. Assume that $g$ satisfies $\left(g_{1}\right)-\left(g_{5}\right)$ and one of the following conditions:

1. $\lambda_{j}<0<\lambda_{j+1}$,

2. $\lambda_{j}=0<\lambda_{j+1}$ and, for some $\delta>0$,

$$
G(x, u) \geq 0 \text { for }|u| \leq \delta,
$$

3. $\lambda_{j}<0=\lambda_{j+1}$ and, for some $\delta>0$,

$$
G(x, u) \leq 0 \text { for }|u| \leq \delta .
$$

If $j \geq 3$, problem (4) has at least three nontrivial solutions. 
We seek critical points of

$$
F(u)=\int_{\Omega} \frac{1}{2}\left(|\nabla u|^{2}+a(x) u^{2}\right)-G(x, u)
$$

on $X=H_{0}^{1}(\Omega)$.

Lemma 3.3. If $g$ satisfies $\left(g_{1}\right),\left(g_{3}\right)-\left(g_{5}\right)$, then, for $\alpha<0$ and $|\alpha|$ sufficiently large,

$$
H_{q}\left(X, F_{\alpha}\right)=0 \quad \forall q \in \mathbb{Z} .
$$

Proof. Let $\tilde{X}=C_{0}^{1}(\bar{\Omega})$ and $\tilde{F}=\left.F\right|_{\tilde{X}}$. By elliptic regularity, $F$ and $\tilde{F}$ have the same critical set. If $F$ does not have any critical values in $\left(\alpha, \alpha^{\prime}\right)$, then $F_{\alpha}$ (respectively $\tilde{F}_{\alpha}$ ) is a strong deformation retract of $\left\{u \in X: F(u)<\alpha^{\prime}\right\}$ (respectively $\left\{u \in \tilde{X}: \tilde{F}(u)<\alpha^{\prime}\right\}$ ) (see Chapter I, Theorem 3.2 and Chapter III, Theorem 1.1 of Chang [5]). Since $\tilde{X}$ is dense in $X$, by a theorem of Palais [12],

$$
H_{q}\left(X,\left\{F<\alpha^{\prime}\right\}\right) \cong H_{q}\left(\tilde{X},\left\{\tilde{F}<\alpha^{\prime}\right\}\right)
$$

Therefore it suffices to prove that, for $\alpha<0$ and $|\alpha|$ large,

$$
H_{q}\left(\tilde{X}, \tilde{F}_{\alpha}\right)=0 \quad \forall q \in \mathbb{Z} .
$$

Let $S^{\infty}=\left\{u \in \tilde{X}:\|u\|_{X}=1\right\}$ be the unit sphere in $\tilde{X}$ and let $S_{+}^{\infty}=$ $\left\{u \in S^{\infty}: u>0\right.$ somewhere $\}$, which is a relatively open subset of $S^{\infty}$, contractible to $\left\{\phi_{1}\right\}$ via $(t, u) \longmapsto \frac{(1-t) u+t \phi_{1}}{\left\|(1-t) u+t \phi_{1}\right\|} t \in[0,1]$. We shall show that $\tilde{F}_{\alpha}$ is homotopy equivalent to $S_{+}^{\infty}$ for $\alpha<0$ and $|\alpha|$ large.

By $\left(g_{3}\right)$ and $\left(g_{5}\right)$,

$$
\begin{gathered}
-C\left(1+u^{2}\right) \leq G(x, u) \leq \frac{1}{2} \lambda_{1} u^{2}+C \text { for } u \leq A, \\
G(x, u) \geq C u^{\mu} \text { for } u \geq A,
\end{gathered}
$$

where $C$ denotes (possibly different) positive constants. Thus for $u \in S_{+}^{\infty}$,

$$
\begin{aligned}
\tilde{F}(t u) & =\frac{1}{2}\left(1+\int_{\Omega} a u^{2}\right) t^{2}-\int_{\Omega} G(x, t u) \\
& \leq C\left(1+t^{2}-t^{\mu} \int_{t u \geq A} u^{\mu}\right)
\end{aligned}
$$

and it follows that

$$
\lim _{t \rightarrow \infty} \tilde{F}(t u)=-\infty \text {. }
$$

On the other hand, in $N=\{u \in \tilde{X}: u \leq 0$ everywhere $\}$, the nonpositive cone in $\tilde{X}$,

$$
\tilde{F}(u) \geq \frac{1}{2} \int_{\Omega}\left(|\nabla u|^{2}+a(x) u^{2}-\lambda_{1} u^{2}\right)-C \geq-C .
$$

By $\left(g_{4}\right)$ and $\left(g_{5}\right)$,

$$
\gamma:=\sup _{\bar{\Omega} \times \mathbb{R}}\left(G(x, u)-\frac{1}{2} u g(x, u)\right)<+\infty .
$$


Thus for $u \in S_{+}^{\infty}$ and $t>0$,

$$
\begin{aligned}
\frac{d}{d t} \tilde{F}(t u) & =\left(1+\int_{\Omega} a u^{2}\right) t-\int_{\Omega} u g(x, t u) \\
& =\frac{2}{t}\left\{\tilde{F}(t u)+\int_{\Omega} G(x, t u)-\frac{1}{2} t u g(x, t u)\right\} \\
& \leq \frac{2}{t}\{\tilde{F}(t u)+\gamma|\Omega|\}<0
\end{aligned}
$$

if $\tilde{F}(t u)<-\gamma|\Omega|$.

Fix $\alpha<\min \left\{\inf _{N} \tilde{F},-\gamma|\Omega|, \inf _{\|u\|<1} \tilde{F}\right\}$. Then it follows that for each $u \in S_{+}^{\infty}$ there exists a unique $T(u) \geq 1$ such that

$$
\tilde{F}(t u)\left\{\begin{array}{l}
>\alpha \text { for } 0 \leq t<T(u) \\
=\alpha \text { for } t=T(u) \\
<\alpha \text { for } t>T(u)
\end{array}\right.
$$

and

$$
\tilde{F}_{\alpha}=\left\{t u: u \in S_{+}^{\infty}, t \geq T(u)\right\} .
$$

By the implicit function theorem, $T \in C\left(S_{+}^{\infty},[1, \infty)\right)$. Hence

$$
\eta(s, t u)= \begin{cases}(1-s) t u+s T(u) u & \text { if } 1 \leq t<T(u) \\ t u & \text { if } t \geq T(u)\end{cases}
$$

defines a strong deformation retraction of $\left\{t u: u \in S_{+}^{\infty}, t \geq 1\right\} \simeq S_{+}^{\infty}$ onto $\tilde{F}_{\alpha}$.

Proof of Theorem 3.2. Since $F\left(-t \phi_{1}\right)<0$ for $t>0$ sufficiently small, by standard arguments, $F$ has a local minimizer $u_{0}$ with $u_{0}<0$,

$$
\operatorname{rank} C_{q}\left(F, u_{0}\right)=\delta_{q 0} \text {. }
$$

Since $\lim _{t \rightarrow \infty} F\left(t \phi_{1}\right)=-\infty$, then $F$ also has a mountain pass point $u_{1}$,

$$
\operatorname{rank} C_{q}\left(F, u_{1}\right)=\delta_{q 1} \text {. }
$$

As in the proof of Theorem 3.1,

$$
C_{j}(F, 0) \neq 0,
$$

so, using Lemma 3.3, $F$ also has a nontrivial critical point $u_{j}$ with either

$$
C_{j+1}\left(F, u_{j}\right) \neq 0 \text { or } C_{j-1}\left(F, u_{j}\right) \neq 0 .
$$

Since $j \geq 3, u_{0}, u_{1}, u_{j}$ are distinct nontrivial solutions of (4).

Finally we give an application of Theorem 2.1 to the problem

$$
\left\{\begin{aligned}
-\Delta u+a(x) u & =\lambda g(u) & & \text { in } \Omega, \\
u & =0 & & \text { on } \partial \Omega
\end{aligned}\right.
$$

where $a \in L^{\infty}(\Omega)$ and $g \in C^{1}(\mathbb{R}, \mathbb{R})$ satisfies

$$
\begin{aligned}
& \left(g_{1}\right): \varlimsup_{\lim _{|u| \rightarrow \infty}} \frac{g(u)}{u}<0, \\
& \left(g_{2}\right): g(0)=g^{\prime}(0)=0 .
\end{aligned}
$$


Theorem 3.4. Assume that $g$ satisfies $\left(g_{1}\right)$, $\left(g_{2}\right)$, and one of the following conditions:

1. $\lambda_{j}<0<\lambda_{j+1}$,

2. $\lambda_{j}=0<\lambda_{j+1}$ and, for some $\delta>0$,

$$
G(u) \geq 0 \text { for }|u| \leq \delta,
$$

3. $\lambda_{j}<0=\lambda_{j+1}$ and, for some $\delta>0$,

$$
G(u) \leq 0 \text { for }|u| \leq \delta .
$$

If $j \geq 3$, problem (5) has at least four nontrivial solutions for every $\lambda$ sufficiently large.

Example 3.5. $g(u)= \pm|u| u-u^{3}$

Remark 3.6. See Brézis and Nirenberg [3] and Li and Willem [9] for at least two nontrivial solutions.

Proof of Theorem 3.4. Since, for $\lambda$ sufficiently large, there is an a priori estimate for the solutions of (5) by the maximum principle, we may also assume that $g(u)=b u$ with $b<0$, for $|u|$ large. Then the functional

$$
F(u)=\int_{\Omega} \frac{1}{2}\left(|\nabla u|^{2}+a u^{2}\right)-\lambda G(u)
$$

is well defined on $X=H_{0}^{1}(\Omega)$, and bounded below and satisfies (PS) for $\lambda$ large.

Since $F\left( \pm t \phi_{1}\right)<0$ for $t>0$ sufficiently small, $F$ has two local minimizers $u_{0}^{ \pm}$with $u_{0}^{-}<0<u_{0}^{+}$,

$$
\operatorname{rank} C_{q}\left(F, u_{0}^{ \pm}\right)=\delta_{q 0} .
$$

Then $F$ also has a mountain pass point $u_{1}$,

$$
\operatorname{rank} C_{q}\left(F, u_{1}\right)=\delta_{q 1} \text {. }
$$

As before,

and, for $\alpha<\inf F$,

$$
C_{j}(F, 0) \neq 0
$$

$$
\operatorname{rank} H_{q}\left(X, F_{\alpha}\right)=\delta_{q 0},
$$

so $F$ has a (fourth) nontrivial critical point $u_{j}$ with either

$$
C_{j+1}\left(F, u_{j}\right) \neq 0 \text { or } C_{j-1}\left(F, u_{j}\right) \neq 0 .
$$

\section{REFERENCES}

1. V. Benci and F. Giannoni, Morse theory for $C^{1}$ functionals and Conley blocks. Topol. Methods Nonlinear Anal. 4 (1994), 365-398.

2. V. Benci and D. Fortunato, Periodic solutions of asymptotically linear dynamical systems. NoDEA Nonlinear Differential Equations Appl. 1 (1994), 267-280.

3. H. Brézis and L. Nirenberg, Remarks on finding critical points. Comm. Pure Appl. Math. XLIV (1991), 939-963.

4. K. C. Chang, S. J. Li, and J. Liu, Remarks on multiple solutions for asymptotically linear elliptic boundary value problems. Topol. Methods Nonlinear Anal. 3 (1994), $43-58$. 
5. K.-C. Chang, Infinite-dimensional Morse theory and multiple solution problems, Progress in Nonlinear Differential Equations and their Applications, Vol. 6, Birkhäuser Boston, Boston, 1993.

6. W. Kryszewski and A. Szulkin, An infinite dimensional Morse theory with applications, Trans. Amer. Math. Soc. 349 (1997), 3181-3234.

7. S. J. Li and J. Q. Liu, An existence theorem for multiple critical points and its application, (Chinese), Kexue Tongbao, 29 (1984), no. 17, 1025-1027, 1984.

8. S. J. Li and J. Q. Liu, Morse theory and asymptotically linear Hamiltonian systems, J. Differential Equations, 78 (1989), 53-73.

9. S. J. Li and M. Willem, Applications of local linking to critical point theory, J. Math. Anal. Appl. 189 (1995), 6-32.

10. J. Liu, The Morse index of a saddle point, Systems Sci. Math. Sci. 2 (1989), 32-39.

11. J. Mawhin and M. Willem, Critical point theory and Hamiltonian systems, volume 74 of Applied Mathematical Sciences, Vol. 74, Springer-Verlag, New York, 1989.

12. R. S. Palais, Homotopy theory of infinite dimensional manifolds, Topology, 5 (1966), $1-16$.

13. Z. Q. Wang, On a superlinear elliptic equation, Ann. Inst. H. Poincar Anal. Non Linéaire, 8 (1991), 43-58.

Department of Mathematics

UNIVERSity OF CALIFORNIA IRVINE

IRvine, CA 92697-3875, USA

E-mail: kperera@math.uci.edu 


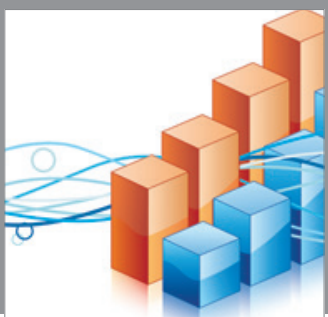

Advances in

Operations Research

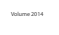

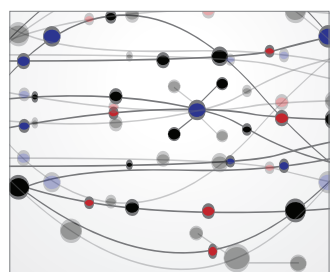

\section{The Scientific} World Journal
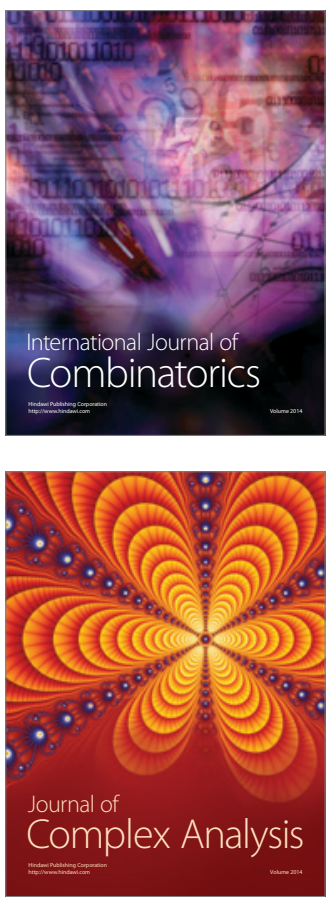

International Journal of

Mathematics and

Mathematical

Sciences
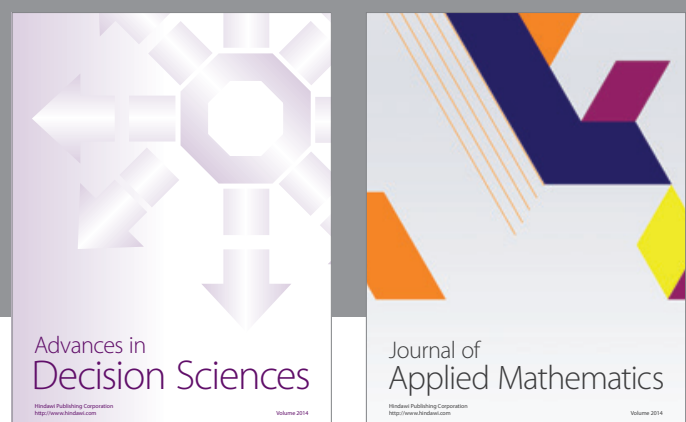

Journal of

Applied Mathematics
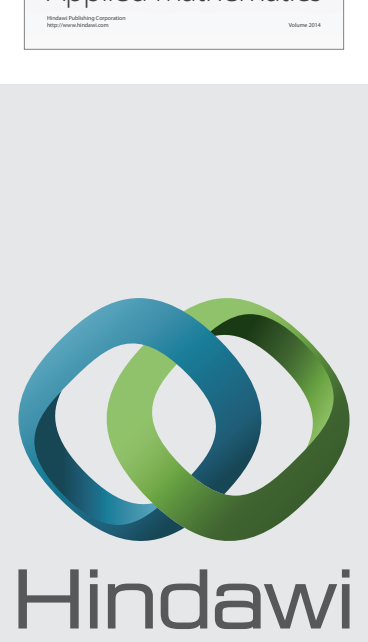

Submit your manuscripts at http://www.hindawi.com
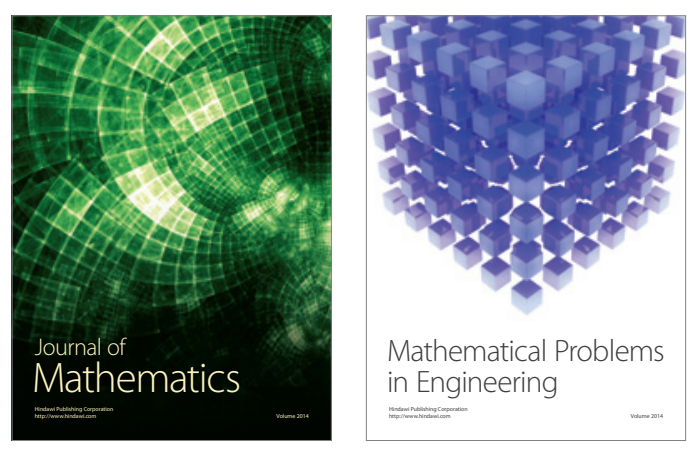

Mathematical Problems in Engineering
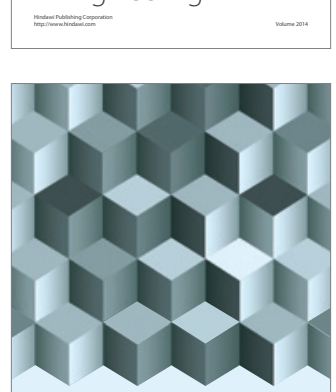

Journal of

Function Spaces
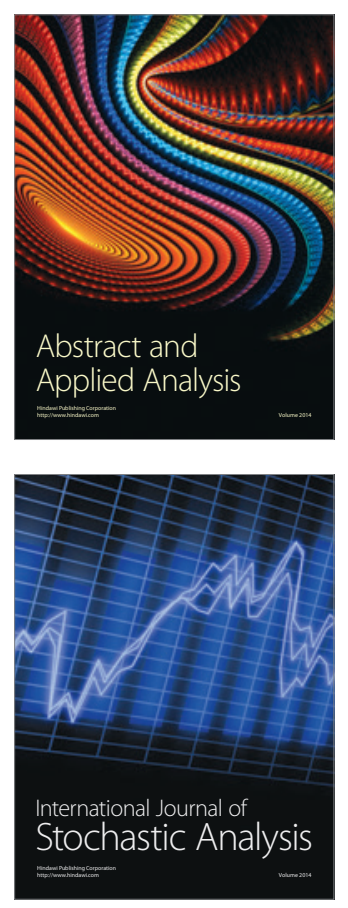

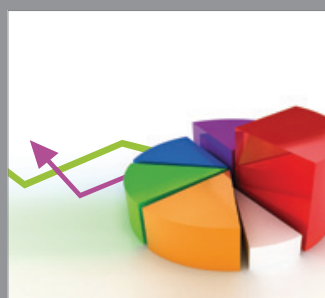

ournal of

Probability and Statistics

Promensencen
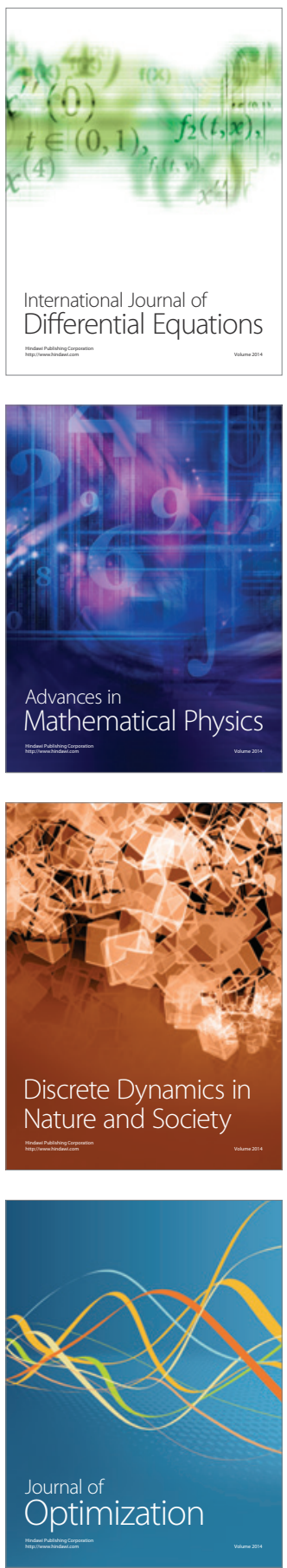